\title{
Prévision de la digestibilité du maïs ensilage par l'étude de la dégradabilité enzymatique des parois cellulaires
}

\author{
R Agneessens 1, P Dardenne 1, P Lecomte 1, P Dujeux 2, T Fourneau 3 \\ Ministère de l'Agriculture, Centre de Recherches Agronomiques de Gembloux, Station de Haute Belgique, \\ 6800 Libramont ; 2lnstitut Supérieur Provincial de Ciney ; Institut Supérieur \\ d'Enseignement Agricole, 4910 Lareid, Belgique
}

\begin{abstract}
Les travaux du Groupe de Travail "Digestibilité", créé pour mieux calculer la valeur énergétique du maïs ensilage, ont démontré qu'aucun des critères "prédictifs", déterminés à la récolte ou au laboratoire, pris individuellement n'expliquait plus d'un tiers des variations de la digestibilité in vivo de la matière organique du maïs ensilage et ce même pour les digestibilités enzymatiques qui ne reflètent isolément que de 20 à $37 \%$ de la variation totale. La combinaison de plusieurs critères (de 3 à 5) permet quant à elle d'obtenir des modèles explicitant jusqu'à $70 \%$ de la variation de la digestibilité observée sur animaux, les modèles les plus précis incluant des critères de composition, de dégradabilité enzymatique et culturaux (conclusions du Groupe de Travail "Digestibilité" septembre 1991). Ces travaux ont également mis en évidence une relation étroite entre la digestibilité de la matière organique et la teneur en parois résiduelles dans les fèces, ce qui a orienté la présente étude.
\end{abstract}

Les essais ont été réalisés sur des échantillons provenant de la banque du Groupe de Travail et pour lesquels les critères de digestibililité in vivo étaient connus. Les parois cellulaires (Neutral Detergent Fiber : NDF) ont été isolées selon la méthode de Goering et Van Soest (1970, Forage Fiber Analyses, Agric Handbook, US Dept Agriculture, 379) en arrêtant la procédure après le séchage du résidu à l'étuve, et la teneur en NDF définie en appliquant le protocole complet (expression sur la matière sèche).

Une aliquote de l'isolat NDF rebroyé et homogénéisé est ensuite hydrolysée dans des creusets filtrants par une enzyme cellulasique (cellulase isolée de Trichoderma virida $\mathrm{BDH}$ 39074 ) pendant 24 heures à $40^{\circ} \mathrm{C}$.

Les résultats de dégradabilité (DNDFo) sont exprimés par rapport à la matière organique en tenant compte de la teneur en cendres résiduelles. Les résultats obtenus, s'ils confirment ceux observés précédemment, c'est à dire une relation relativement faible entre la DMO in vivo et la teneur en NDF, mettent en évidence une relation significative entre la DMO et la dégradabilité enzymatique des parois (DNDFo), ainsi qu'une corrélation quasi nulle entre la quantité et la digestibilité de ces parois.

La combinaison des deux paramètres caractérisant les parois permet de définir un modèle d'estimation de la digestibilité in vivo de la matière organique tout au moins aussi précis que le meilleur modèle retenu lors des études précédentes; ce modèle est défini dans le tableau ci-dessous.

En conclusion, l'étude de la teneur en parois cellulaires et de la digestibilité enzymatique de ces parois devrait permettre une estimation de la digestibilité in vivo de la matière organique du maïs ensilage précise et facilement réalisable en laboratoire, sans tenir compte d'autres paramètres.

$\begin{array}{lll}\text { Modèle } & \text { DMO }=49,40-(0,58475 \times \mathrm{NDF})+(0,53531 \times \mathrm{DNDFo}) \\ & \text { Ecart type coefficients : } & \text { NDF : } 0,056 \\ & & \\ \mathrm{~N} & 84 & \\ \mathrm{R} & 0,89 & \\ \text { RSD } & 1,66 & \end{array}$

